\title{
veterinary specialists
}

\section{Effect of pre-medication drugs on cardiovascular parameters during anaesthesia and on speed of recovery of patients undergoing daily general anaesthesia for radiotherapy.}

Emma Fordham, Christopher Scudder, Sarah L. Mason

Southfields Veterinary Specialists, Laindon, UK

emma.fordham@southfields.co.uk

\section{Objectives}

To develop a protocol for safe pre-medication of patients undergoing general anaesthesia for radiotherapy.

\section{Method}

A nurse-led observational study using client-owned dogs prescribed radiotherapy for treatment of anal sac adenocarcinoma. Pre-medications were randomly assigned; either $0.02 \mathrm{mg} / \mathrm{kg}$ buprenorphine, $0.2 \mathrm{mg} / \mathrm{kg}$ methadone or $0.2 \mathrm{mg} / \mathrm{kg}$ butorphanol. Cardiovascular parameters, mean isoflurane usage, extubation time and total recovery time were recorded.

A repeated measures MANOVA was used to determine differences between cardiovascular parameters data between groups, and repeated measures ANOVA tests for mean isoflurane usage, extubation time and full recovery time. $\mathrm{P}<0.05$ was significant. Post-hoc paired t-tests with Bonferroni correction were performed where appropriate.
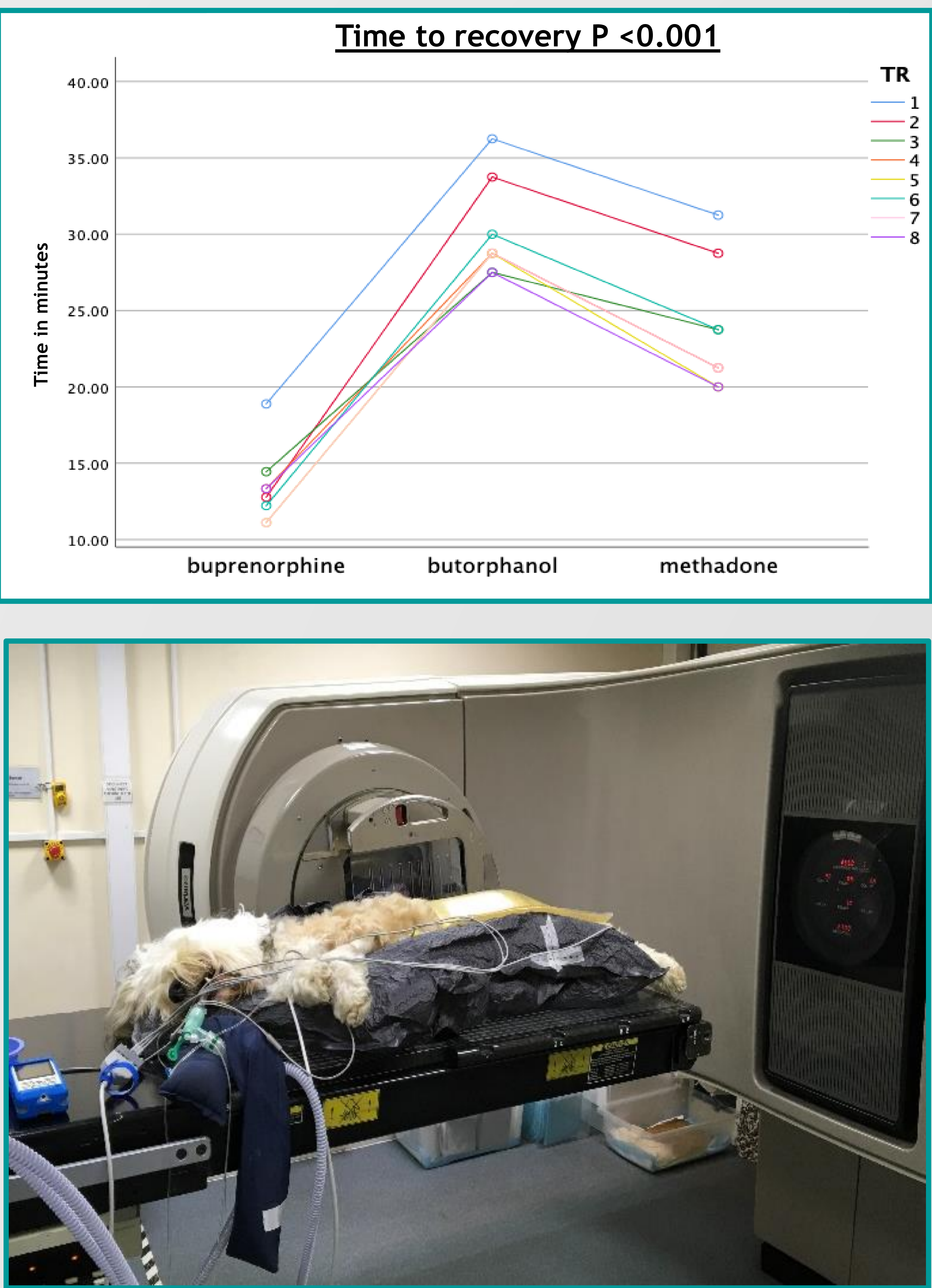

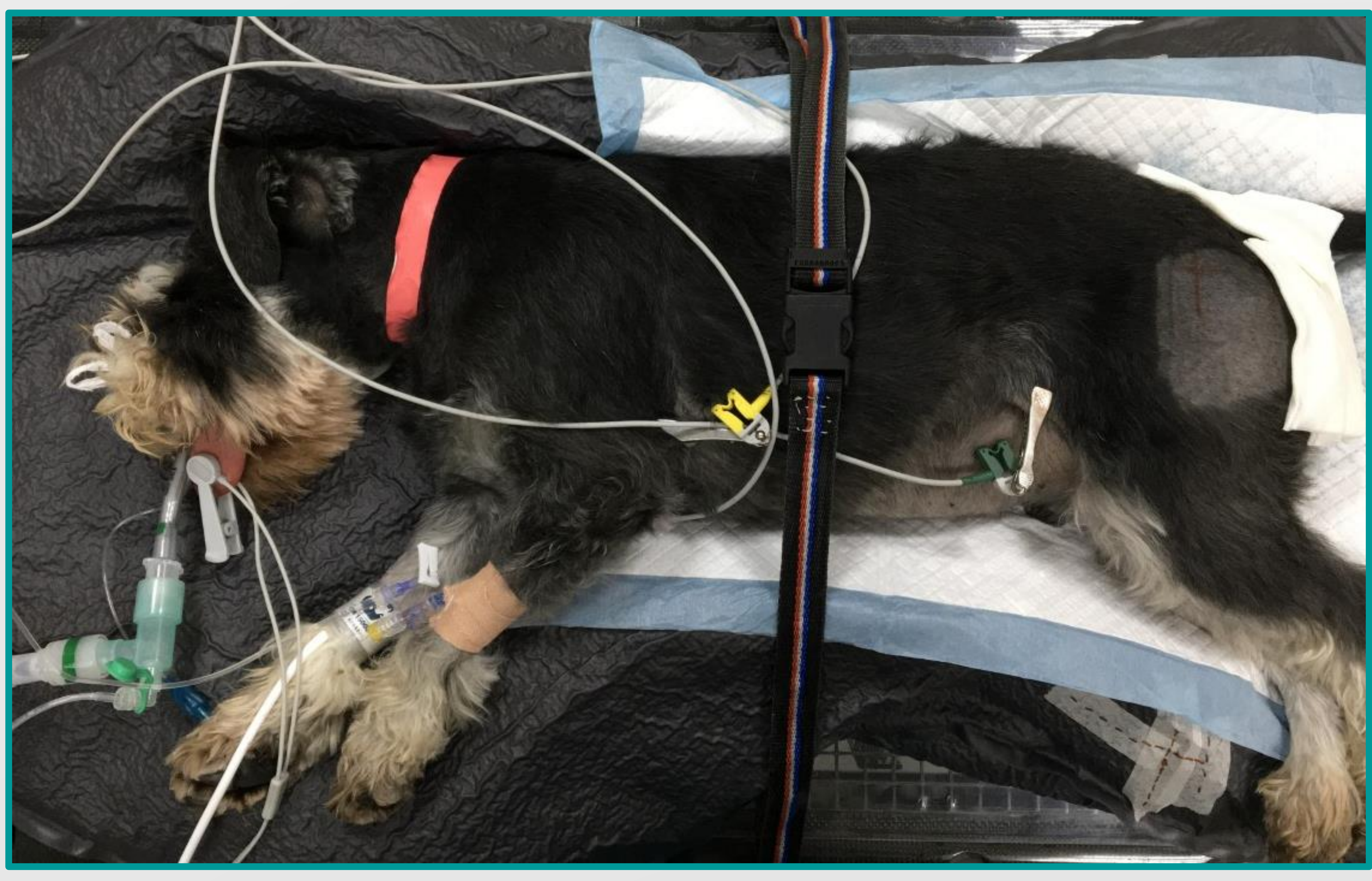

Results

Twenty-four dogs were included, with eight dogs per drug group. There was no difference of cardiovascular parameters between groups $(F=2.045, p=0.140)$. There was a difference of lowest mean isoflurane usage between groups (Wilks Lambda 0.236 $F(7,15) 6.85, p=0.001$ ), with butorphanol resulting in highest usage (butorphanol vs buprenorphine mean difference (M.D.) $0.9 \%, p<0.001$, butorphanol vs methadone M.D 0.6\%, $p<0.001$ ). For extubation time, there was a significant difference between groups (Wilks Lambda $0.167 \mathrm{~F}(7,15) 10.67$, $\mathrm{p}<0.001$ ), with buprenorphine associated with shortest extubation times (buprenorphine vs butorphanol M.D. -3.1 minutes (mins) $p<0.001$, buprenorphine vs methadone M.D. -2.3 mins $p<0.001)$. There was a significant difference of total recovery time (Wilks Lambda $0.241 F(7,15) 6.75, p=0.001)$, with buprenorphine associated with shortest recovery time (buprenorphine vs butorphanol M.D. -16.3 mins $p<0.001$, buprenorphine vs methadone M.D. -9.9 mins $\mathrm{p}<0.001)$.

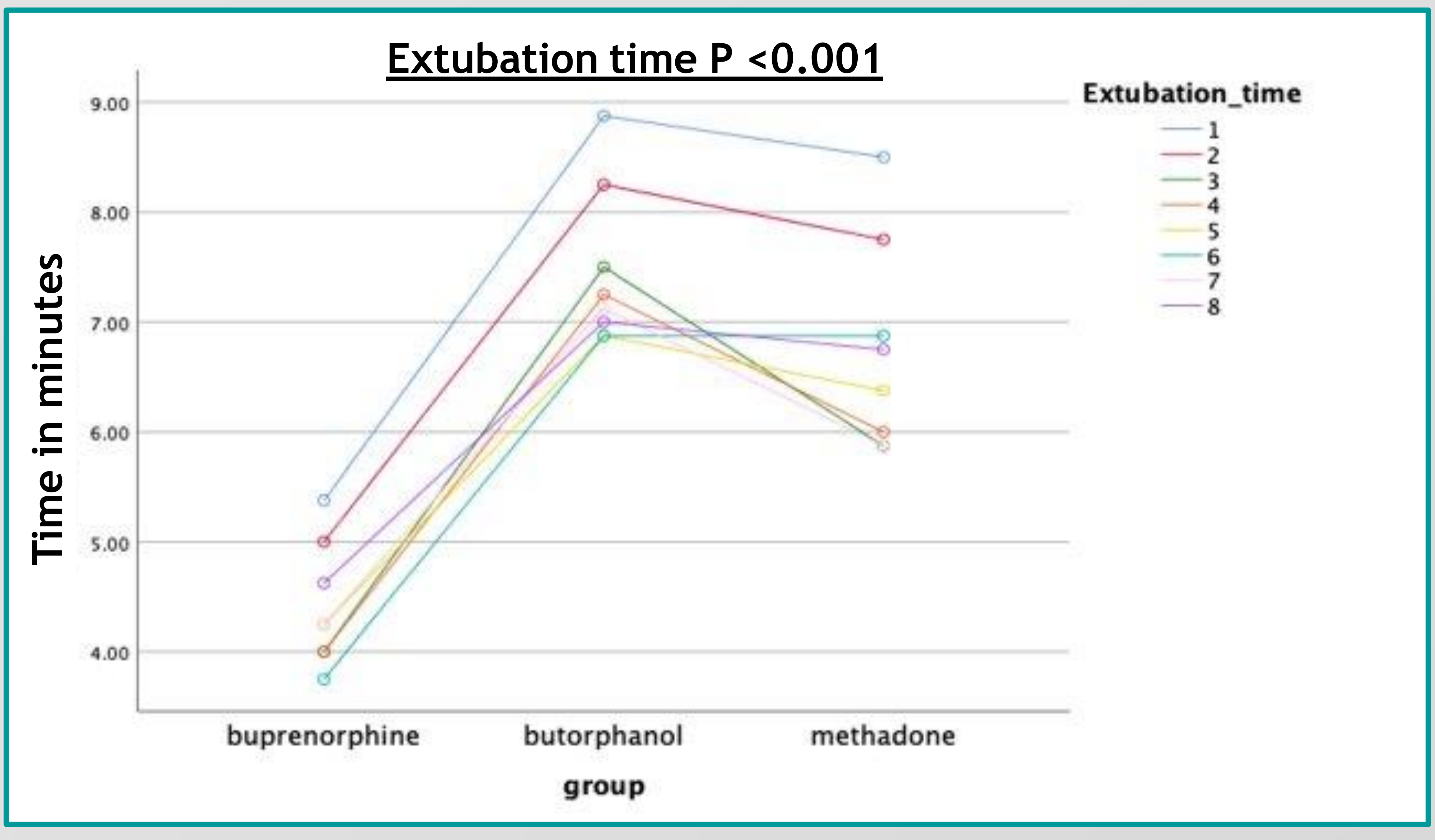

Clinical Significance

Buprenorphine reduced isoflurane usage and recovery times and appears to be the drug of choice for these patients. 